\title{
Simulación y optimización para dimensionar la flota de vehículos en operaciones logísticas de abastecimiento-distribución
}

\author{
Simulation and optimization for fleet size determination \\ in the supply-distribution logistic operations \\ Yasel José Costa Salas ${ }^{1} \quad$ Néstor Jaime Castaño Pérez ${ }^{2}$ \\ Recibido 3 de junio de 2014, aceptado 6 de octubre de 2014 \\ Received: June 3, 2014 Accepted: October 6, 2014
}

\begin{abstract}
RESUMEN
En este trabajo se muestra el empleo simultáneo de la simulación y optimización en la planeación de operaciones logísticas, específicamente para apoyar la toma de decisiones relacionadas con la cantidad de medios de transporte a utilizar en el proceso de abastecimiento-distribución de mercancías. El estudio se desarrolla para una compañía cubana dedicada a la transportación de bienes y servicios de almacenamiento. La aplicación de las herramientas propuestas permitió sugerir el número óptimo de camiones a utilizar, considerando minimizar el tiempo ocioso de los camiones y maximizar el nivel de servicio al cliente. Los resultados experimentales muestran una mejora significativa en la reducción del plazo de entrega y en el aumento de la cantidad de pedidos satisfechos para los clientes de la compañía.
\end{abstract}

Palabras clave: Tamaño de flota, simulación, optimización, tiempo ocioso, nivel de servicio al cliente.

\begin{abstract}
In this paper, a simultaneous use of simulation and optimization techniques for the logistic operation planning is presented, specifically aimed to support the decision making processes related with fleet size determination in the supply-distribution process. The study is developed for a Cuban company dedicated to the transportation of goods and warehousing services. The implementation of the proposed tools enabled suggesting the optimal number of trucks to be used, considering minimization of the idle time of trucks and maximization of the customer service level. Experimental results show a significant improvement regarding the lead time reduction and the increment of order fulfillment for the customers of the company.
\end{abstract}

Keywords: Fleet size, simulation, optimization, idle time, customer service level.

\section{INTRODUCCIÓN}

El servicio al cliente es considerado la actividad rectora dentro de la planeación logística para cualquier empresa [1-2]. Dicha actividad constituye el punto de partida para el diseño de las demás actividades en el proceso de planeación [3-4]. El objetivo de una compañía siempre está enfocado a proporcionar bienes o servicios a un cliente indicado. Sin embargo, ningún producto o servicio tiene una utilidad real si no está en posesión del cliente, en el momento en que lo necesita y en el lugar donde el cliente pueda acceder a él. En este sentido, las decisiones de Enrutamiento de Vehículos (EV) resultan el centro de atención para lograr tales propósitos. Estas decisiones tienen estrecho vínculo con una larga familia de problemas combinatorios, estos reflejan los fenómenos de entrega o recogida de personas

1 University of Applied Sciences. Universidad de Manizales. Carrera 9 No 19-03. Código Postal: 17001. Manizales, Colombia. E-mail: yasel.costa@umanizales.edu.co

2 Universidad de Manizales. Carrera 9 No 19-03. Código Postal: 17001. Manizales, Colombia. E-mail: njcastanop@gmail.com 
y mercancías usando una flota de vehículos [5]. A grandes rasgos, un problema de EV involucra dos decisiones de gran complejidad: la determinación del tamaño de la flota de vehículos y las rutas "adecuadas" a seguir por dichos vehículos [5-6]. No obstante, se plantea que determinar el tamaño óptimo de la flota resulta la más compleja matemáticamente, además de tener el mayor impacto económico en la toma de decisiones [7].

Múltiples estrategias de solución han sido propuestas en la literatura especializada con vista a determinar el tamaño óptimo de una flota de vehículos. Sin embargo, todas pueden resumirse en dos categorías predominantes: métodos exactos y aproximados [8]. Dentro de la primera categoría, métodos exactos, la literatura muestra varios enfoques de solución, los que se han aplicado exitosamente a problemas de pequeña escala (escala expresada en cantidad de clientes a visitar). Por ejemplo, la Programación Dinámica [9], Relajación Lagrangiana [10], Ramificación y Poda (Branch and Bound) [11] y Generación de Columnas (Column Generation Algorithm) [1213] figuran dentro de los enfoques exactos más empleados. Desafortunadamente la complejidad computacional de este tipo de problema combinatorio provoca un crecimiento no polinomial (complejidad no polinomialmente acotada, NP según sus siglas en idioma inglés), este se hace poco factible para problemas de dimensiones reales.

Los algoritmos aproximados aparecen en la literatura científica como una vía alternativa de solución para la complejidad de los problemas de EV, en particular para la determinación del tamaño "apropiado" de la flota de vehículos. A diferencia de los algoritmos exactos, el proceso de búsqueda de un algoritmo aproximado no se desarrolla de forma exhaustiva [14]. Por tal razón, es poco probable que estos algoritmos encuentren una solución óptima del problema bajo estudio al solo examinar parte de su espacio de posibles soluciones. Sin embargo, logran encontrar soluciones de muy buena calidad [15] en tiempos de ejecución razonables [16], características valiosas desde el punto de vista práctico para el decisor. En esta categoría de métodos matemáticos, las heurísticas, metaheurísticas y los algoritmos de aproximación componen las diversas familias de algoritmos más aplicados. Entre las heurísticas clásicas aplicadas al dimensionamiento de flota se encuentran la heurística del Barrido (Sweep) [17], usada para flotas heterogéneas (vehículos con diferente capacidad) y la heurística de inserción (Insertion Algorithms) [18].

Las metaheurísticas han recibido la mayor atención en la última década. En este campo resulta impresionante el número de investigaciones desarrolladas para EV. Aunque el dimensionamiento de las flotas, dentro de los problemas de EV, no ha tenido similar atención que la determinación de rutas, la cantidad de algoritmos metaheurísticos aplicados a la problemática sigue siendo significativa. En la Tabla 1 se muestran algunas de las metaheurísticas empleadas en la temática, así como la denominación a la que pertenecen y su contexto de aplicación.

A pesar de los múltiples algoritmos aproximados, aplicados al dimensionamiento de la flota de vehículos, los algoritmos de aproximación y en particular las técnicas basadas en simulación no han tenido similar tratamiento. Solo en escasas ocasiones se reportan soluciones basadas en simulación para problemáticas relacionas con el EV. En este sentido pueden ser citados problemas muy particulares, como logística de distribución [19-20], problemas de tráfico [21] y gestión integrada inventariosestrategias de transportación [22].

Considerando los aspectos antes descritos, en la presente investigación se propone el uso de la simulación de eventos discretos y la optimización de escenarios como alternativa de solución al problema de dimensionamiento de flotas de vehículos. En la investigación se aborda la problemática mencionada para una empresa cubana, esta se especializa en servicios de transportación y almacenamiento. $\mathrm{La}$ dirección de esta compañía precisaba conocer el monto de inversión necesario para la adquisición de camiones con el objetivo de incrementar el nivel de servicio al cliente en el proceso de abastecimientodistribución de mercancías. Las complejidades del problema, provocadas por la combinación de las actividades de abastecimiento y distribución (crecimiento exponencial en el costo computacional [6] a medida que crecen las actividades de distribución y abastecimiento), así como el carácter estocástico de las variables involucradas (demanda para la distribución, cantidad para el abastecimiento y el índice de rotura de los medios de transporte), condujeron a la necesidad de utilizar el enfoque propuesto basado en simulación de eventos discretos. 
Tabla 1. Metaheurísticas empleadas para la determinación de la flota de vehículos.

\begin{tabular}{|c|c|c|c|}
\hline Denominación & Autores & Metaheurística & Contexto de aplicación \\
\hline \multirow{4}{*}{$\begin{array}{l}\text { Trayectoria } \\
\text { simple }\end{array}$} & [23] & $\begin{array}{l}\text { Búsqueda tabú } \\
\text { (Tabu Search) }\end{array}$ & $\begin{array}{l}\text { EV con ventanas de tiempo y flota } \\
\text { heterogénea }\end{array}$ \\
\hline & [24] & $\begin{array}{l}\text { Recocido simulado } \\
\text { (Simulated Annealing) }\end{array}$ & Transportación de mercancías por ferrocarril \\
\hline & [25] & $\begin{array}{l}\text { Búsqueda en vecindad } \\
\text { Variable (Variable } \\
\text { Neighborhood Search) }\end{array}$ & EV con flota heterogénea \\
\hline & [13] & $\begin{array}{l}\text { Búsqueda local iterada } \\
\text { (Iterated Local Search) }\end{array}$ & EV en logística humanitaria \\
\hline \multirow[b]{2}{*}{$\begin{array}{l}\text { Estrategias } \\
\text { evolutivas }\end{array}$} & [26] & $\begin{array}{l}\text { Algoritmo genético } \\
\text { (Genetic Algorithm) }\end{array}$ & Distribución en camiones de carga \\
\hline & [27] & $\begin{array}{l}\text { Algoritmo evolutivo } \\
\text { (Knowledge-based } \\
\text { Evolutionary Algorithm) }\end{array}$ & $\begin{array}{l}\text { Entorno multiobjetivo y con ventanas de } \\
\text { tiempo }\end{array}$ \\
\hline \multirow[b]{2}{*}{$\begin{array}{l}\text { Inteligencia } \\
\text { colectiva }\end{array}$} & [28] & $\begin{array}{l}\text { Algoritmos de hormigas } \\
\text { (Ant Algorithms) }\end{array}$ & $\begin{array}{l}\text { EV con flota heterogénea, ventanas de } \\
\text { tiempo y múltiples productos }\end{array}$ \\
\hline & [16] & $\begin{array}{l}\text { Optimización basada en } \\
\text { enjambre de partículas } \\
\text { (Particle Swarm } \\
\text { Optimization) }\end{array}$ & $\begin{array}{l}\text { EV para carros eléctricos de tipo } \\
\text { heterogéneos (dependiendo del tipo de } \\
\text { batería) }\end{array}$ \\
\hline
\end{tabular}

La propuesta de la presente investigación estuvo basada en la metodología de [29], la que está compuesta de los pasos siguientes: 1) definición de objetivos, alcance y requerimientos; 2) definición de objetivos para la toma de decisiones; 3 ) recolección y análisis de los datos del sistema; 4) construcción del modelo de simulación; 5) validación del modelo; 6) conducción del experimento de optimización; 7) presentación de resultados.

Los resultados experimentales obtenidos proporcionaron suficientes evidencias para demostrar las ventajas del uso de la simulación-optimización. La investigación concluye con la propuesta del escenario más apropiado para la compañía, este presenta una mejora significativa respecto del tiempo de entregas y el total de pedidos atendidos

\section{METODOLOGÍA}

Para conducir el proceso de optimización de la flota se siguió un procedimiento sencillo de seis pasos (ver Figura 1), el que ilustra, de forma adecuada, la naturaleza iterativa de este tipo de proceso. Una breve descripción del contenido de cada paso, perteneciente a la metodología, es presentada en seguida.

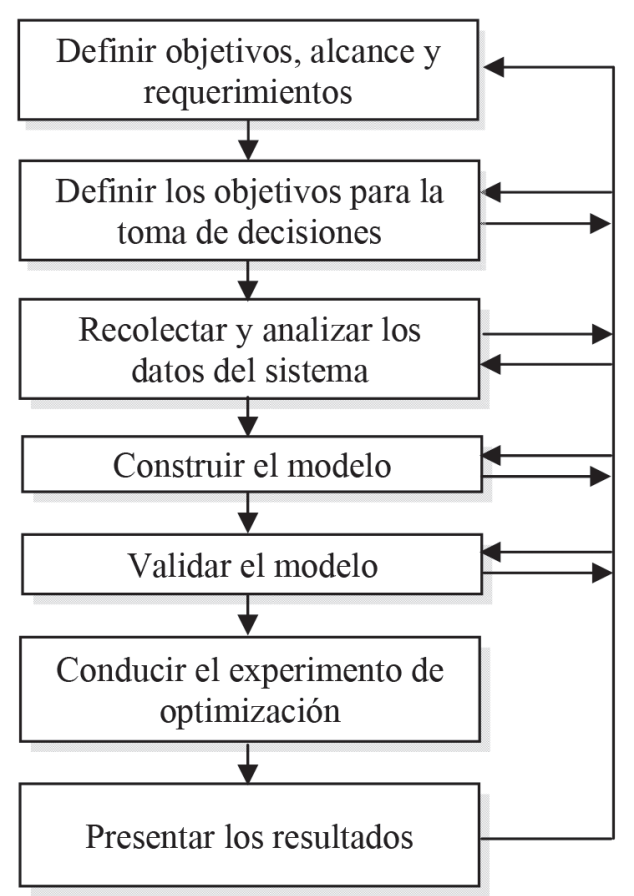

Figura 1. Proceso iterativo de la Simulación. Fuente: [29]. 


\section{Pasos de la metodología}

1) Definir objetivos, alcance y requerimientos. Los objetivos deciden el resto de los pasos e influyen en el diseño del experimento. Deben ser realistas, tener un impacto potencial alto, específicos, alcanzables, debido a las limitaciones del estudio (tiempo y recursos) y cuantificables. El alcance del proyecto es una especificación que ayuda a entender lo que la simulación debe incluir y excluir. Mientras que los requerimientos del sistema implican los recursos necesarios para desarrollar la simulación (principalmente computacionales).

2) Definir los objetivos para la toma de decisiones. Este paso conduce al estudio de las necesidades reales de la empresa objeto de estudio en donde se aplica la simulación. El diseño de los objetivos dependerá de la significativa incidencia de los mismos para las métricas de desempeño.

3) Recolectar y analizar los datos del sistema. El paso implica la colección, registro, conversión, transmisión y manipulación de los datos del sistema. Su resultado es un modelo mental de cómo el sistema está configurado. La aplicación de este paso requiere la correcta definición de los elementos del sistema, desarrollar la descripción de las operaciones (ejemplo, utilizando diagramas de flujo) y tomar datos y analizarlos estadísticamente (cálculo de tamaños de muestra, pruebas de bondad de ajuste, etc.)

4) Construir el modelo de simulación. La finalidad para la creación del modelo consiste en lograr el balance entre el realismo y finalidad del mismo. El modelo puede construirse demasiado simplificado (fácil de programar e interpretar, pero puede dar resultados no válidos) o muy detallado, lo que puede ser difícil de programar e implementar.

5) Validar el modelo. En este paso se verifica si el modelo de simulación refleja correctamente el modelo conceptual o mental (buscar errores, inconsistencias y omisiones) y si el modelo conceptual refleja de modo correcto la realidad (comparación de datos reales de desempeño con datos obtenidos en la simulación, por lo general esto implica una vinculación con la estadística en la aplicación de pruebas de hipótesis).
6) Conducir el experimento de optimización. Los resultados del modelo de simulación generan múltiples alternativas de solución. Este paso propone desarrollar todas las acciones necesarias para la creación de un experimento de optimización (implica la definición de objetivos, datos de entrada y número de escenarios de simulación) que tendrá, como espacio de combinaciones, las posibles alternativas de solución.

7) Presentar los resultados. Consiste en proporcionar los resultados más relevantes para la toma de decisiones o para posteriores análisis que complementen el estudio. Es recomendable, por lo general, el empleo de tablas, estadísticos descriptivos y otras formas de presentación de la información con vista a hacer fácil su comprensión.

\section{RESULTADOS}

En esta sección se detalla, para la empresa objeto de estudio, cada uno de los pasos del procedimiento enunciado anteriormente. Para la simulación del sistema estudiado se propone el uso del software Promodel 7.0 y para la optimización de escenarios de simulación se propone el módulo SimRunner 3.2.0.1. La elección de ambas implementaciones computacionales estuvo basada en su flexibilidad y simplicidad para la representación de problemas, analíticamente complejos, como la planeación del tamaño de la flota de vehículos.

\section{Paso 1. Definición del objetivo, alcance y requerimientos: En consulta con la gerencia de la compañía objeto de estudio (Almacenes Universales S.A., Sucursal Villa Clara), se definió que el objetivo o propósito para conducir el estudio de simulación sería encontrar la cantidad óptima de camiones a emplear en el proceso de abastecimiento-distribución de mercancías que minimice el tiempo ocioso de los camiones y maximice el nivel de servicio al cliente, expresado este último en las variables plazo de entrega y cantidad de pedidos satisfechos.}

La compañía, además del almacenamiento, incluye entre sus servicios el de distribución de mercancías del cliente Los Portales S.A., para ello cuenta con cinco camiones. Estos dos servicios constituyen el alcance de esta investigación y en la Figura 2 se 


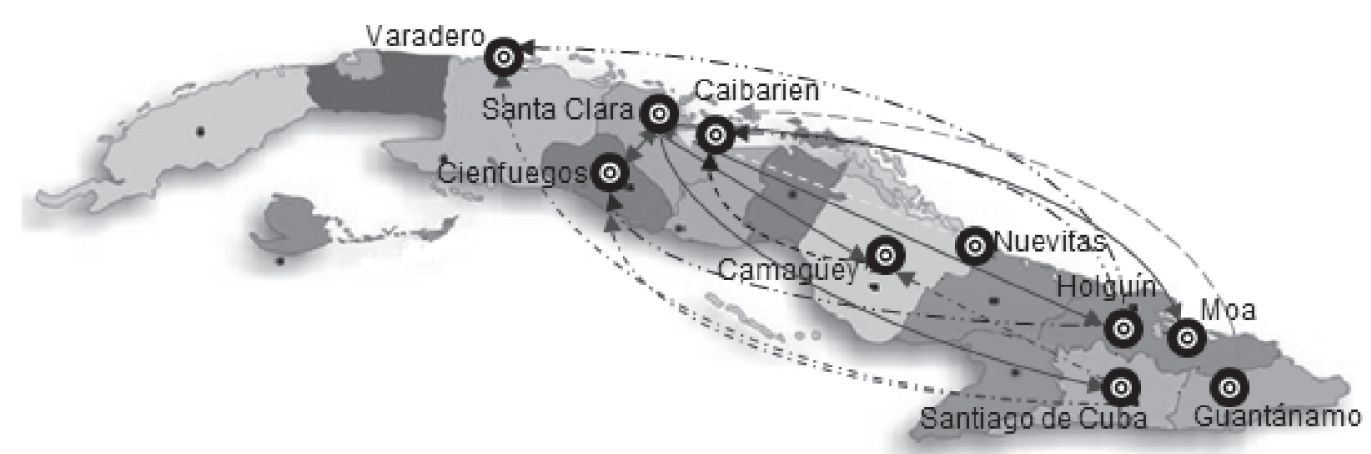

Figura 2. Red de distribución y abastecimiento (Los Portales S.A) desde los puntos de destino.

muestran los orígenes y destinos en estos procesos de transportación de mercancías.

Estos puntos (orígenes y destinos) constituirán las locaciones del modelo y cuando coincida que una ciudad es origen y destino, deberán considerarse para esta dos locaciones separadas. Las locaciones deben considerarse con capacidad infinita. En cuanto a las entidades, estas pueden ser consideradas de manera general como mercancías, diferenciándolas por su locación de procedencia; una orden de entrega a transportar equivale a una mercancía. Se considerarán las distancias entre ciudades y las velocidades con carga y sin carga de los camiones, así como los tiempos de carga y descarga. Así mismo, es importante tomar en cuenta las fallas de los camiones y el tiempo dedicado a la reparación y mantenimiento de estos. Para la experimentación a partir del módulo SimRunner 7.0 se considerarán, por restricciones de presupuesto para la inversión, cuatro escenarios (con cinco, seis, siete y ocho camiones, respectivamente).

\section{Paso 2. Definición de objetivos para la toma de} decisiones: Este paso se abordó teniendo en cuenta las necesidades de la empresa en cuanto al uso de los camiones en la transportación de mercancías. Se le brindó asesoramiento a la gerencia (responsable de tomar la decisión) en cuanto a que los objetivos que se seleccionaran debían ser independientes unos de otros, no redundantes y establecer cierto nivel de conflicto en el proceso de toma de decisiones. Después de una sesión de trabajo se logró definir como objetivos para seleccionar el número óptimo de camiones a utilizar en el proceso de transportación, los siguientes: 1) minimizar el tiempo de inactividad de los camiones; 2) maximizar la cantidad de salidas (mercancías transportadas) y 3) minimizar el tiempo promedio de las mercancías en el sistema.

Paso 3. Recolección y análisis de los datos del sistema: Durante este paso se realizó un análisis del sistema de datos necesarios para construir el modelo. Para ello se dividieron los datos necesarios en tres categorías: estructurales, operacionales y numéricos. En cuanto a los estructurales se consideraron la cantidad de locaciones, recursos y entidades, así como las redes de caminos. El análisis de los datos operacionales permitió explicar cómo opera el sistema, es decir, cuándo, dónde y cómo tienen lugar las actividades.

El análisis de los datos anteriores permitió definir las necesidades de datos numéricos, estos se tomaron de acuerdo con la experiencia de los choferes, a los registros históricos de la empresa y a la observación personal del investigador. Para ello se calcularon los tamaños de muestra necesarios y se procesaron, en los casos que se requería, en el software BestFit 4.5.2, para determinar la distribución de mejor ajuste utilizando la prueba Kolmogorov-Smirnov. En la Tabla 2 se muestra un resumen de los resultados obtenidos para las variables tiempo entre arribos de las solicitudes. Otros datos tomados fueron los tiempos de transportación entre los diferentes puntos así como los de carga y descarga.

Paso 4. Construcción del modelo de simulación: Para la construcción del modelo se realizó un estudio detallado de la operación del sistema actual. Se consideraron quince (15) locaciones, siete (7) tipos de entidades, un (1) tipo recurso (camión), una (1) red con diecisiete (17) segmentos de caminos. En la Figura 3 se muestra, como ejemplo, una de las 
Tabla 2. Resultados prueba Kolmogorov-Smirnov en las variables tiempo entre arribos de solicitudes.

\begin{tabular}{|l|c|c|c|}
\hline \multicolumn{1}{|c|}{$\mathbf{A}$} & Desde & $\begin{array}{c}\text { Distribución de mejor ajuste } \\
\text { sus parámetros (en días) }\end{array}$ & $p$-value (Prueba $K$-S) \\
\hline \multirow{4}{*}{ Santa Clara } & Santiago de Cuba & $\mathrm{E}(1,8)$ & 0,056 \\
\cline { 2 - 4 } & Moa & $\mathrm{L}(1 ; 0,1)$ & 0,120 \\
\cline { 2 - 4 } & Holguín & $\mathrm{N}(1,2 ; 0,2)$ & 0,067 \\
\cline { 2 - 4 } & Camagüey & $\mathrm{N}(1 ; 0,2)$ & 0,087 \\
\cline { 2 - 4 } & Cienfuegos & $\mathrm{N}(1 ; 0,1)$ & 0,118 \\
\hline Nuevitas & Santa Clara & $\mathrm{E}(2,2)$ & 0,076 \\
\hline Cienfuegos & Santa Clara & $\mathrm{E}(1,5)$ & 0,089 \\
\hline Camagüey & Caibarién & $\mathrm{N}(2 ; 0,5)$ & 0,151 \\
\hline Guantánamo & Caibarién & $\mathrm{E}(3,1)$ & 0,097 \\
\hline Santiago de Cuba & - & $\mathrm{E}(2,3)$ & 0,087 \\
\hline Holguín & - & $\mathrm{N}(2 ; 0,4)$ & 0,134 \\
\hline
\end{tabular}

ventanas definidas en el Promodel 7.0 durante la construcción del modelo.

Paso 5. Validación del modelo de simulación: Una vez construido el modelo y previo a su validación se pasó a determinar el número de réplicas necesarias y el warm-up period o periodo de tiempo necesario para que el sistema alcanzara estabilidad. Para ello se estableció un error de estimación del $1 \%$, un nivel de confianza del $95 \%$ y periodos de dos días cada uno. Utilizando el módulo SimRunner y específicamente el método de Welch Moving Average [30] se pudo determinar que para obtener estimaciones de acuerdo con los parámetros fijados se precisan de 28 réplicas (ver Figura 4).

Además se obtuvo que a partir del cuarto periodo (ocho días) el sistema comenzaba a estabilizarse (ver Figura 4). Con estos resultados y para validar el modelo se realizó una corrida de 100 días con 100 réplicas; se tomaron, posteriormente, los resultados de las variables tiempo de entrega a Camagüey, Santiago de Cuba, Holguín, Moa y Cienfuegos y se compararon estadísticamente con la misma cantidad de datos de valores históricos de estas variables. Esta comparación se realizó a partir de

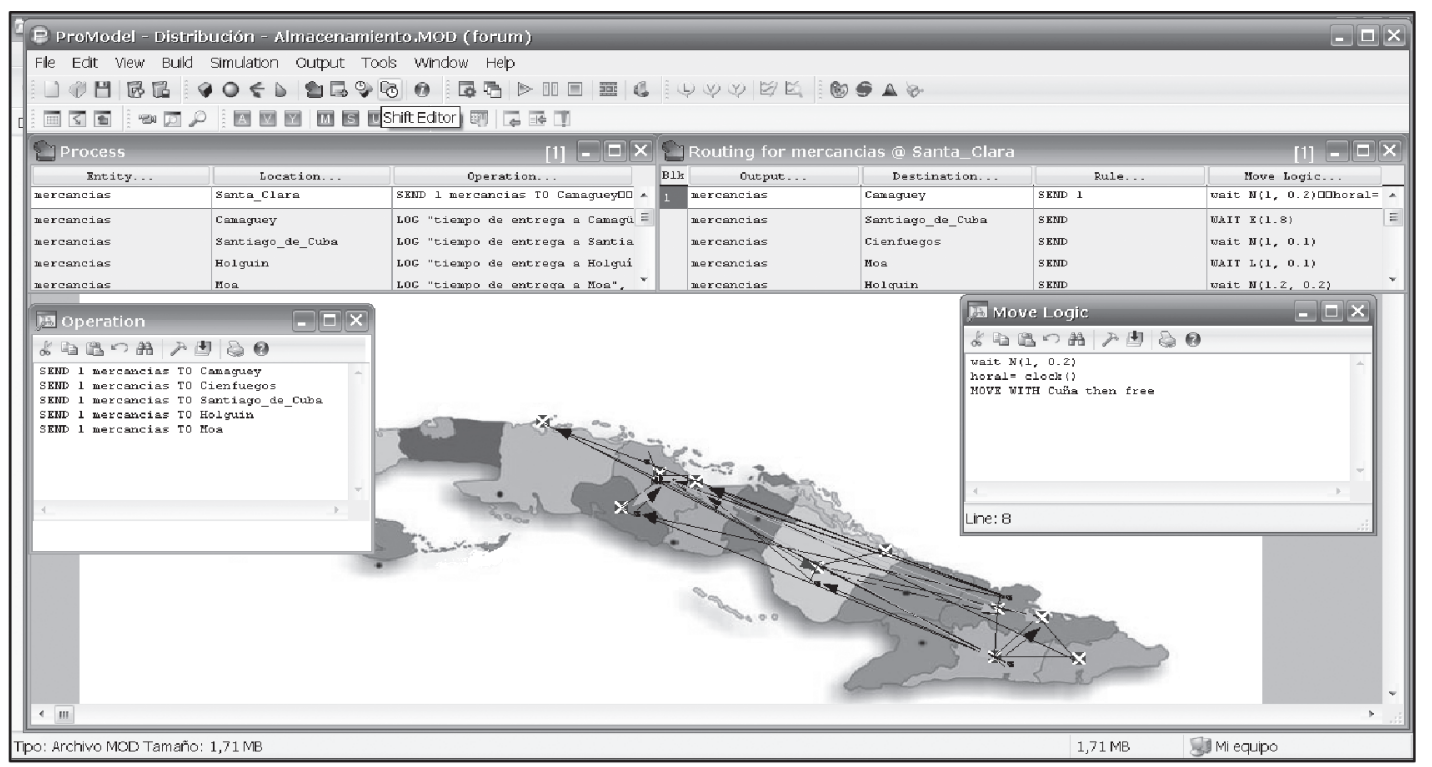

Figura 3. Definición del procesamiento en la construcción del modelo.

Fuente: Promodel 7.0. 


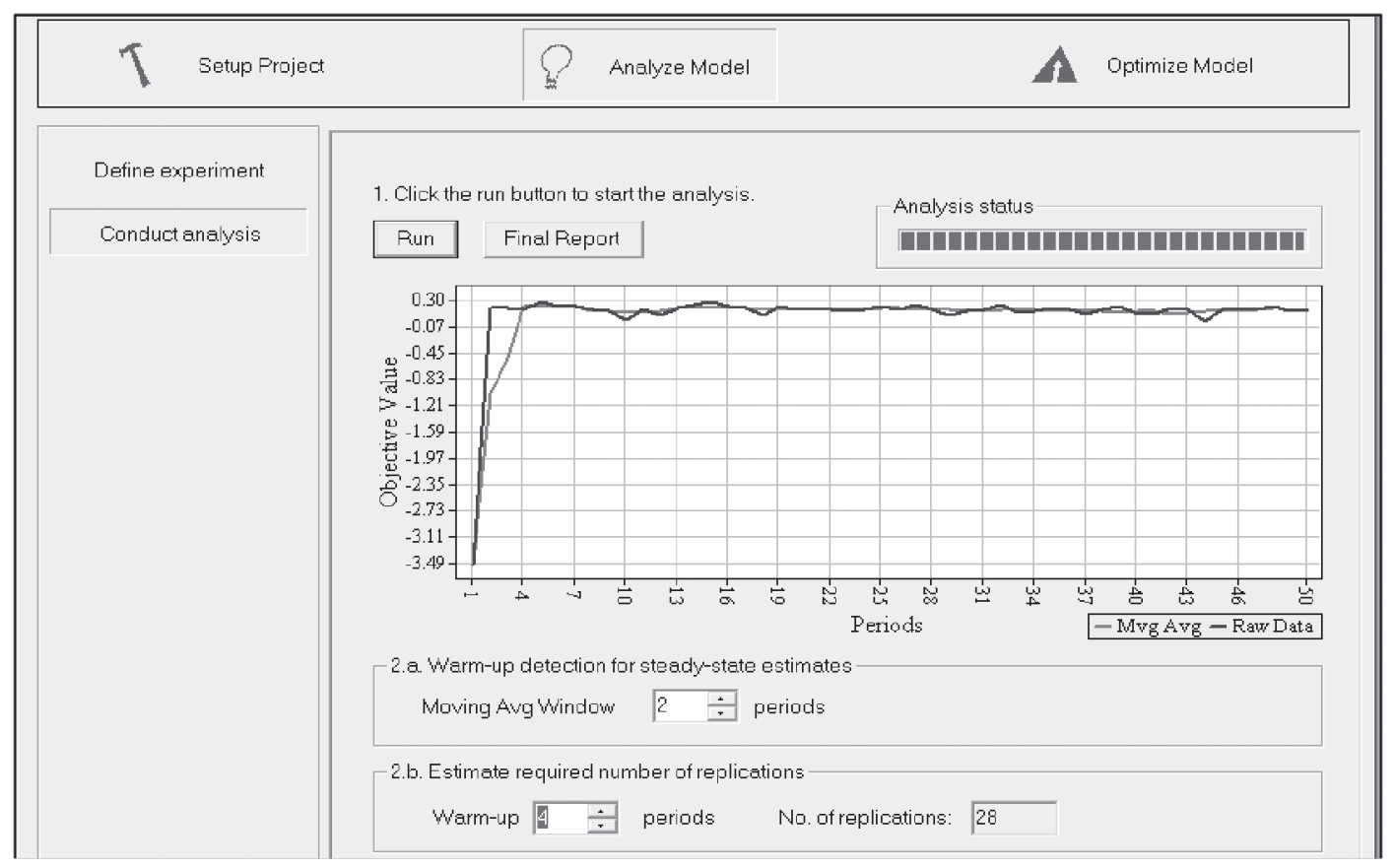

Figura 4. Determinación del número de réplicas y del Warm-up period.

Fuente: SimRunner 3.2.0.1.

Tabla 3. Comparación estadística de las medias reales y simuladas, variables tiempo de entrega.

\begin{tabular}{|l|c|c|c|c|c|}
\hline \multirow{2}{*}{} & \multicolumn{6}{|c|}{$\begin{array}{c}\text { Test de muestra independiente } \\
\end{array}$} & $\begin{array}{c}\text { Levene para igualdad } \\
\text { de varianzas }\end{array}$ & \multicolumn{2}{c|}{ t-test para igualdad de masas } \\
\cline { 2 - 6 } & $\mathbf{F}$ & Sig. & t & df & Sig. (2-tailed) \\
\hline TE-Cam & 0,317 & 0,574 & 0,510 & 198 & 0,611 \\
\hline TE-Sant & 0,020 & 0,889 & 0,671 & 198 & 0,503 \\
\hline TE-Holg & 4,645 & 0,032 & $-0,301$ & 183,846 & 0,764 \\
\hline TE-Moa & 1,889 & 0,171 & 0,079 & 198 & 0,937 \\
\hline TE-Cienf & 0,494 & 0,483 & 0,991 & 198 & 0,323 \\
\hline
\end{tabular}

la prueba $T$ de Student para el contraste de medias, previo análisis del cumplimiento de sus requisitos, y permitió validar el modelo (ver Tabla 3).

Paso 6. Conducción del experimento de optimización: Una vez validado el modelo se procedió a su utilización con fines de optimización y así cumplir con los objetivos fijados al inicio de la investigación. Se consideraron 14 elementos dentro de la función objetivo (todos con igual importancia), relacionados estos con la cantidad de solicitudes servidas (Total Exits), el tiempo promedio en el sistema de dichas solicitudes (Average Time in System) y el porcentaje de tiempo ocioso de los camiones (\% Idle).
En cuanto a los factores de entrada (Input Factors), como ya se había planteado en puntos anteriores, se incluyó el análisis de la cantidad de camiones entre cinco y ocho. La ejecución de los cuatro experimentos en el módulo de optimización del SimRunner 3.2.0.1 (ver Figuras 5 y 6) permitió sugerir que la empresa debía incrementar su parque de camiones hasta siete.

Paso 7. Presentación de los resultados: Con el objetivo de brindar información relevante a la empresa para que esta desarrolle los análisis económicos necesarios en el proceso de toma de decisiones acerca de la cantidad de camiones a comprar, se 


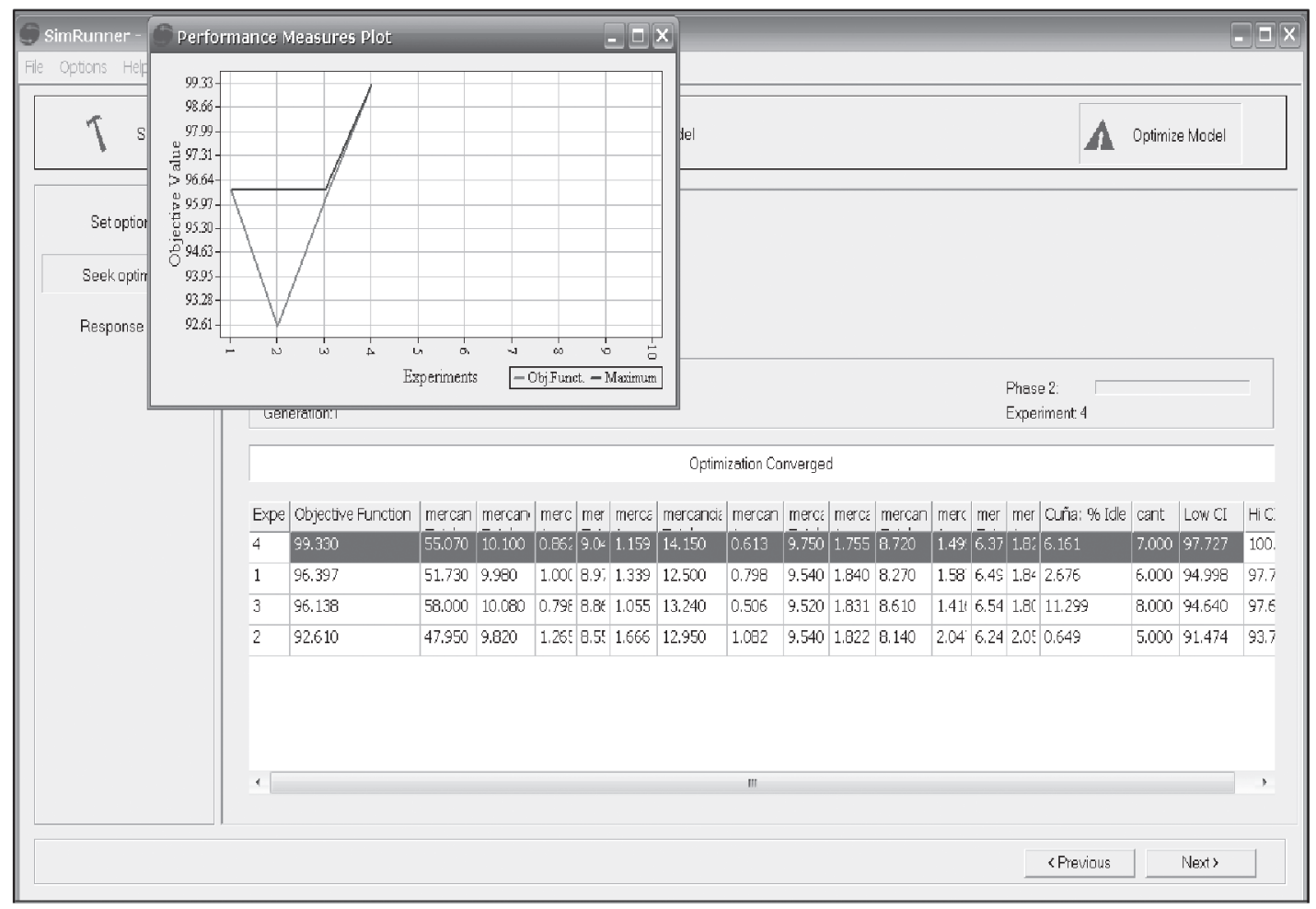

Figura 5. Valor de funciones objetivo para los cuatro experimentos diseñados en SimRunner 3.2.0.1.

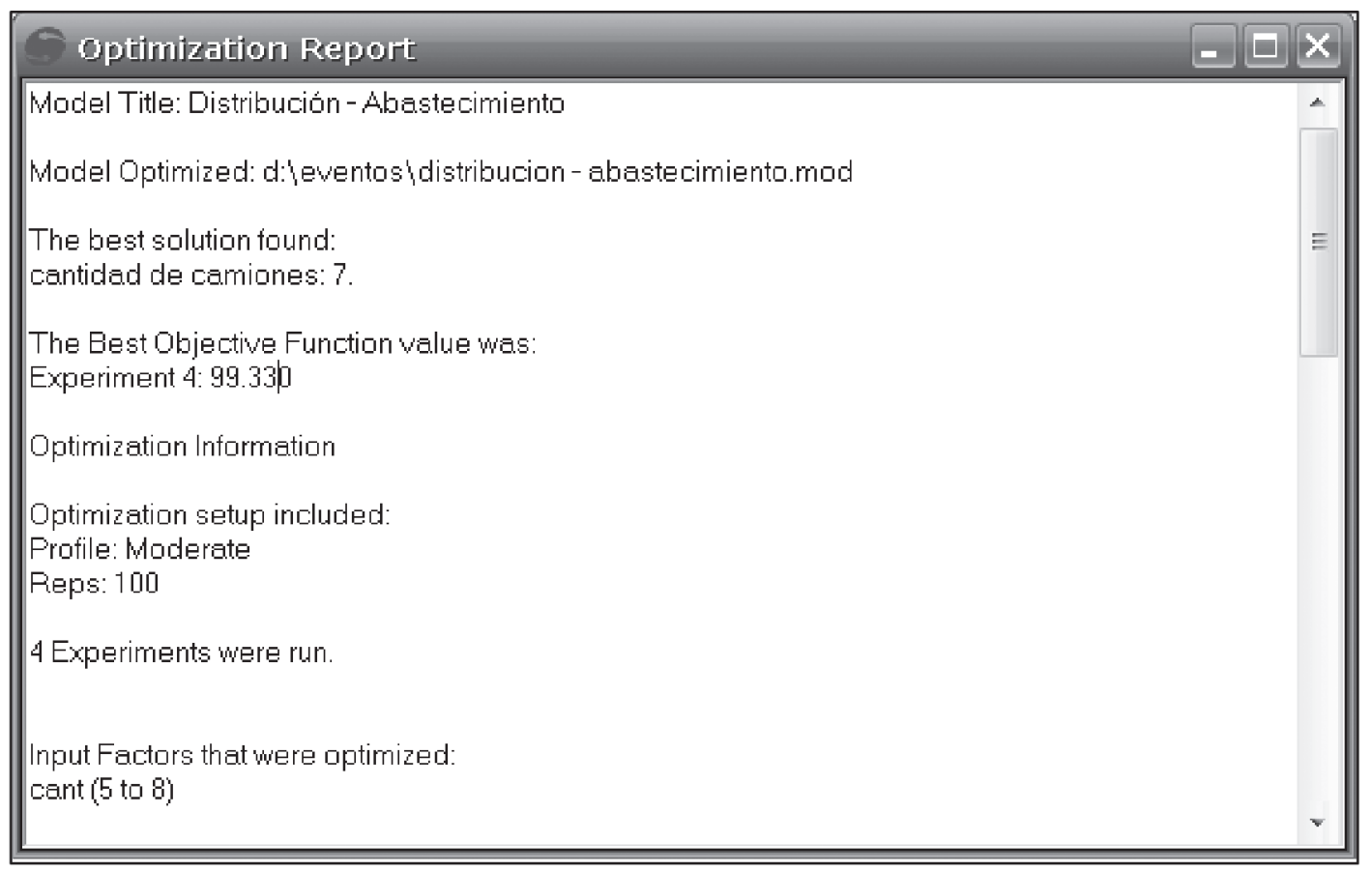

Figura 6. Reporte de optimización para el problema objeto de estudio.

Fuente: SimRunner 3.2.0.1. 
Tabla 4. Comparación entre los escenarios actual (cinco camiones) y propuesto (siete camiones)

\begin{tabular}{|l|l|c|c|c|}
\hline \multicolumn{2}{|c|}{ Variables } & \multicolumn{2}{c|}{ Escenarios } & \multirow{2}{*}{ Diferencia } \\
\cline { 2 - 4 } & Actual & Propuesto & \\
\hline \multicolumn{2}{|c|}{ Total de solicitudes atendidas } & 496 & 534 & 38 \\
\hline \% de tiempo ocioso promedio de los camiones & 0,0093 & 1,605 & 1,5957 \\
\hline \multirow{4}{*}{$\begin{array}{l}\text { Tiempo } \\
\text { de entrega } \\
\text { (días) }\end{array}$} & Santa Clara & 1,1449 & 0,9058 & $-0,2391$ \\
\cline { 2 - 4 } desde: & Nuevitas & 3,9537 & 1,6178 & $-2,3359$ \\
\cline { 2 - 4 } & Cienfuegos & 2,3978 & 0,9886 & $-1,4092$ \\
\cline { 2 - 4 } & Camagüey & 3,0348 & 1,1899 & $-1,8449$ \\
\cline { 2 - 4 } & Guantánamo & 4,1626 & 2,1099 & $-2,0527$ \\
\cline { 2 - 4 } & Holguín & 6,2518 & 2,0058 & $-4,2460$ \\
\cline { 2 - 4 } & Santiago de Cuba & 9,1786 & 2,0052 & $-7,1734$ \\
\hline
\end{tabular}

presentó una comparación, a partir de la simulación de 100 días con 100 réplicas, de dos escenarios: el actual (con cinco camiones) y el propuesto (con siete camiones).

En la Tabla 4 se puede observar la mejora que se lograría en las variables total de solicitudes atendidas y tiempo de entrega. Como promedio, en cien días, se atenderían 38 solicitudes (esto implica una utilidad mínima de USD 1.600 por orden) más y el tiempo de entrega se reduciría en 2,76 días, con una reducción significativa para las mercancías que se transportan desde Santiago de Cuba y Holguín. Con un comportamiento negativo el porcentaje de tiempo ocioso de los camiones se incrementaría en $1,5957 \%$, lo que no resulta preocupante si se tiene en cuenta que esta cifra significaría, aproximadamente, 12 minutos por día de trabajo.

\section{CONCLUSIONES}

La investigación permitió demostrar las potencialidades de la combinación "simulación y optimización" como herramienta de apoyo al proceso de toma de decisiones en la empresa. Los resultados experimentales obtenidos, luego de aplicar la metodología, posibilitan arribar a las conclusiones siguientes: 1) La simulación constituye una técnica de extraordinario valor para la toma de decisiones en contexto estocástico. 2) La conformación de escenarios de simulación reduce la complejidad en los problemas combinatorios, facilitando la posterior aplicación de algoritmos de optimización para encontrar soluciones de buena calidad en tiempos de ejecución razonables. 3) La aplicación simultánea de la simulación y la optimización proporciona un carácter proactivo a la toma de decisiones, en particular cuando se formulan decisiones para el problema analizado sin realizar transformaciones acerca del sistema real. 4) La empresa objeto de estudio debe considerar el redimensionamiento de su flota de camiones, la cual operando con siete (7) de éstos mejora significativamente el nivel de servicio al cliente. 5) La compañía contará con la información necesaria para llevar a cabo de forma objetiva y confiable un proceso de análisis de factibilidad económica.

\section{AGRADECIMIENTOS}

El autor desea agradecer el acceso a los datos e informaciones de la empresa Almacenes Universales S.A. (Cuba), de igual forma el apoyo brindado por su gerencia durante el desarrollo de la investigación.

\section{REFERENCIAS}

[1] A. Baghalian, S. Rezapour and R.Z. Farahani. "Robust supply chain network design with service level against disruptions and demand uncertainties: A real-life case". European Journal of Operational Research. Vol. 227, pp.199-215. 2013. ISSN: 0377-2217. DOI: 10.1016/j.ejor.2012.12.017.

[2] U.D. Kumar. "Supply Chains with Service Level Agreements". In: U. Ramanathan, R. Ramanathan (Eds.) Supply Chain Strategies, Issues and Models, Springer. Vol. 1, pp. 209219. London. ISBN: 978-1-4471-5351-1. 2014.

[3] M.Y.A. Rawwas and K.N.S. Iyer. "How do small firms possibly survive? A comparison 
study of marketing skills and logistics infrastructure of small and large wholesalers". International Business Review. Vol. 22, pp. 687-698. 2013. ISSN: 0969-5931. DOI: 10.1016/j.ibusrev.2012.10.003.

[4] A. Sohal and S. Rahman. "Use of Third Party Logistics Services: An Asia-Pacific Perspective". In: J.H. Bookbinder (Ed.) Handbook of Global Logistics, Springer. Vol. 181, pp. 45-67. New York. ISBN: 9781-4419-6131-0. 2013.

[5] B.L. Golden, S. Raghavan and E.A. Wasil. "The Vehicle Routing Problem: Latest Advances and New Challenges: latest advances and new challenges". Springer, pp. 54-67. New York. ISBN: 9780387777788. 2008.

[6] Y.J. Costa Salas, R. Abreu Ledón, C. Machado Oses y N.I. Coello Machado. "Asistencia decisional en el proceso de optimización para el ruteo de vehículos. Revista Ingeniería Industrial. Vol. 31, pp.1-7. 2010. ISSN: 1815-5936.

[7] A. Hoff, H. Andersson, M. Christiansen, G. Hasle and A. Løkketangen. "Industrial aspects and literature survey: Fleet composition and routing". Computers \& Operations Research. Vol. 37, pp. 2041-2061. 2010. ISSN: 03050548. DOI: $10.1016 /$ j.cor.2010.03.015.

[8] G. Laporte. "The vehicle routing problem: An overview of exact and approximate algorithms". European Journal of Operational Research. Vol. 59, pp. 345358. 1992. ISSN: 0377-2217. DOI: 10.1016/0377-2217(92)90192-C.

[9] M. Desrochers, J. Desrosiers and M. Solomon. "A New Optimization Algorithm for the Vehicle Routing Problem with Time Windows". Operations Research. Vol. 40, pp. 342-354. 1992. ISSN: 1526-5463. DOI: 10.1287/opre.40.2.342.

[10] J. Desrosiers, M. Sauvé and F. Soumis. "Lagrangian Relaxation Methods for Solving the Minimum Fleet Size Multiple Traveling Salesman Problem with Time Windows". Management Science. Vol. 34, pp. 10051022. 1988. ISSN: 1526-5501. DOI: $10.1287 /$ mnsc.34.8.1005.

[11] G. Ulusoy. "The fleet size and mix problem for capacitated arc routing". European Journal of Operational Research. Vol. 22, pp. 329-337. 1985. ISSN: 0377-2217. DOI: 10.1016/0377-2217(85)90252-8.

[12] E. Choi and D.-W. Tcha. "A column generation approach to the heterogeneous fleet vehicle routing problem". Computers \& Operations Research. Vol. 34, pp. 2080-2095. 2007. ISSN: 0305-0548. DOI: 10.1016/j. cor.2005.08.002.

[13] H.M. Afsar, C. Prins and A.C. Santos. "Exact and heuristic algorithms for solving the generalized vehicle routing problem with flexible fleet size". International Transactions in Operational Research. Vol. 21, pp. 153175. 2014. ISSN: 1475-3995. DOI: 10.1111/ itor.12041.

[14] W. Meihua, T. Xuhong, C. Shan and W. Shumin. "Hybrid Ant Colony Optimization Algorithm for Two Echelon Vehicle Routing Problem". Procedia Engineering. Vol. 15, pp. 3361-3365. 2011. ISSN: 1877-7058. DOI: 10.1016/j.proeng.2011.08.630.

[15] A.D. López-Sánchez, A.G. Hernández-Díaz, D. Vigo, R. Caballero and J. Molina. "A multi-start algorithm for a balanced real-world Open Vehicle Routing Problem". European Journal of Operational Research. Vol. 238, pp. 104-113. 2014. ISSN: 0377-2217. DOI: 10.1016/j.ejor.2014.04.008.

[16] A.A. Juan, J. Goentzel and T. Bektaş. "Routing fleets with multiple driving ranges: Is it possible to use greener fleet configurations?". Applied Soft Computing. Vol. 21, pp. 84-94. 2014. ISSN: 1568-4946. DOI: $10.1016 / \mathrm{j}$. asoc.2014.03.012.

[17] J. Renaud and F.F. Boctor. "A sweep-based algorithm for the fleet size and mix vehicle routing problem". European Journal of Operational Research. Vol. 140, pp. 618-628. 2002. ISSN: 0377-2217I. DOI: $10.1016 /$ S0377-2217(01)00237-5.

[18] N. Perrier, A. Langevin and J.F. Campbell. "A survey of models and algorithms for winter road maintenance. Part IV: Vehicle routing and fleet sizing for plowing and snow disposal". Computers \& Operations Research. Vol. 34, pp. 258-294. 2007. ISSN: 0305-0548. DOI: 10.1016/j.cor.2005.05.008.

[19] C.H. White. "Distribution logistics in the process industries: establishing railcar requirements". Proceedings of the 28th conference on Winter simulation, IEEE 
Computer Society. pp. 1367-1372. Coronado, California, USA. 1996.

[20] M.A. Guerrero Hernández y A.F. Henriques Librantz. "Simulación de eventos discretos de la cadena logística de exportación de commodities. Ingeniare. Revista chilena de ingeniería. Vol. $22 \mathrm{~N}^{\mathrm{o}} 2$, pp. 257-262. 2014. ISSN: 0718-3305.DOI: $10.4067 /$ S0718-3052014000200011.

[21] M. Garzón and G. Mendez. "Improving service of access on a logistics platform". Proceedings of the 2010 Spring Simulation Multiconference, Society for Computer Simulation International. pp. 1-7. Orlando, Florida, USA. 2010.

[22] L. EunSu and K. Farahmand. "Simulation of a base stock inventory management system integrated with transportation strategies of a logistic network". Simulation Conference (WSC) Proceedings of the 2010 Winter. pp. 1934-1945. 2010.

[23] J. Jiang, K.M. Ng, K.L. Poh and K.M. Teo. "Vehicle routing problem with a heterogeneous fleet and time windows". Expert Systems with Applications. Vol. 41, pp. 3748-3760. 2014. ISSN: 0957-4174. DOI: 10.1016/j.eswa.2013.11.029.

[24] M. Yaghini and Z. Khandaghabadi. "A hybrid metaheuristic algorithm for dynamic rail car fleet sizing problem". Applied Mathematical Modelling. Vol. 37, pp. 41274138. 2013. ISSN: 0307-904X. DOI: 10.1016/j.apm.2012.09.013.
[25] S.-C. Liu and H.-J. Lu. "A hybrid heuristic method for the fleet size and mix vehicle routing problem". Journal of Industrial and Production Engineering. Vol. 30, pp. 181-189. 2013. ISSN: 2168-1015. DOI: 10.1080/21681015.2013.800163.

[26] S.-H. Huang, T.-H. Yang and C.-H. Tang. "Fleet size determination for a truckload distribution center". Journal of Advanced Transportation. Paper in press. 2012. ISSN: 2042-3195. DOI: 10.1002/atr.1180.

[27] T.-C. Chiang and W.-H. Hsu. "A knowledgebased evolutionary algorithm for the multiobjective vehicle routing problem with time windows". Computers \& Operations Research. Vol. 45, pp. 25-37. 2014. ISSN: 0305-0548. DOI: 10.1016/j. cor.2013.11.014.

[28] J. Cruz, C. Paternina-Arboleda, V. Cantillo and J. Montoya-Torres. "A two-pheromone trail ant colony system - tabu search approach for the heterogeneous vehicle routing problem with time windows and multiple products". Journal of Heuristics. Vol. 19, pp. 233-252. 2013. ISSN: 1381-1231. DOI: $10.1007 /$ s10732-011-9184-0.

[29] C. Harrel, B.K. Ghosh and R. Bowden. "Simulation using Promodel". McGrawHill Higher Education. $N^{\circ} 3$. USA. ISSN: 0073112119. 2012.

[30] A.M. Law and W.D. Kelton. "Simulation modeling and analysis". McGraw-Hill. USA. ISSN: 9780070592926. 2000. 